\title{
Two-photon interferences with degenerate and nondegenerate paired photons
}

\author{
Chang Liu, ${ }^{1}$ J. F. Chen, ${ }^{1}$ Shanchao Zhang, ${ }^{1}$ Shuyu Zhou, ${ }^{1}$ Yoon-Ho Kim, ${ }^{2}$ M. M. T. Loy, ${ }^{1}$ \\ G. K. L. Wong, ${ }^{1}$ and Shengwang $\mathrm{Du}^{1, *}$ \\ ${ }^{1}$ Department of Physics, The Hong Kong University of Science and Technology, Clear Water Bay, Kowloon, Hong Kong, China \\ ${ }^{2}$ Department of Physics, Pohang University of Science and Technology (POSTECH), Pohang 790-784, Korea
}

(Received 7 September 2011; published 13 February 2012)

\begin{abstract}
We generate narrow-band frequency-tunable entangled photon pairs from spontaneous four-wave mixing in three-level cold atoms and study their two-photon quantum interference after a beam splitter. We find that the path-exchange symmetry plays a more important role in the Hong-Ou-Mandel interference than the temporal or frequency indistinguishability, and observe coalescence interference for both degenerate and nondegenerate photons. We also observe a quantum beat in the same experimental setup using either slow or fast detectors.
\end{abstract}

DOI: 10.1103/PhysRevA.85.021803

PACS number(s): 42.50.Ar, 42.50.Dv, 03.67.Mn, 42.50.St

Quantum interference between single photons is one of the most important physical mechanisms for realizing linear optical quantum computation and information processing $[1,2]$. When two photons meet on a beam splitter (BS), their fourth-order interference can be observed as a coincidence correlation between two single-photon detectors, each placed at the output ports of the BS. In the well-known Hong-OuMandel (HOM) interference [3,4], it was found that two identical photons coalesce in the BS and go together into the same output port. This two-photon quantum interference effect has been confirmed from paired photons in spontaneous parametric down-conversion [3], four-wave mixing [5], and single photons from independent sources [6,7]. In these experiments, the indistinguishability between the two photons is the key for observing the HOM effect where the detectors are slow as compared to the bandwidth of the photons. When the two photons are in different frequencies, the two-photon interference can be measured as a quantum beat in time domain by two fast detectors [8,9].

It was later realized that the HOM effect is not the interference between two individual photons, but the interference resulting from different two-photon Feynman paths $[10,11]$. Therefore, indistinguishability of the two photons (which holds in the early experiments) may not be the necessary condition for observing the two-photon interference. This explains why the HOM dip is immune from the groupvelocity dispersion [12]. The HOM interference has also been demonstrated using photons with distinguishable polarizations [13]. The HOM effect with photons of different colors was also predicted [13-15]. By adjusting the phase difference between the two-photon Feynman paths, one can even change the HOM dip into a peak using an entangled photon source $[10,11,13]$. In this Rapid Communication, we report an experimental demonstration of the HOM interference with nondegenerate photon pairs. We find that, while the photons may be distinguishable in their temporal and/or frequency degrees of freedom, the indistinguishable pathways from the spatialmode exchange symmetry leads to the HOM interference for both the degenerate and nondegenerate paired photons in our right-angle spontaneous four-wave mixing (SFWM)

\footnotetext{
*dusw@ust.hk
}

configuration. In addition to directly observing the quantum beat with fast detectors, we also show that the quantum beat between nondegenerate photons can be measured in the HOM setup by two slow detectors (without filtering at the detectors as in Refs. [16,17]).

The experimental setup and atomic energy-level configuration are shown in Figs. 1(a) and 1(b). We produce narrow-band Stokes $\left(\omega_{s}\right)$ and anti-Stokes $\left(\omega_{a s}\right)$ paired photons using SFWM in cold atoms in the presence of counterpropagating pump $\left(\omega_{p}\right)$ and coupling $\left(\omega_{c}\right)$ laser beams [18,19]. Both pump and coupling lasers are operated at a $\mathrm{Rb} D_{2}$ line $780 \mathrm{~nm}$. The pump laser is blue detuned from the transition $|1\rangle \rightarrow|3\rangle$ by $\Delta_{p}$. The coupling laser, on resonance with the transition $|2\rangle \rightarrow|3\rangle$, renders the medium transparent for the anti-Stokes photons. The central-frequency difference between the Stokes and anti-Stokes photons is determined by $\Delta \omega=\Delta_{12}-\Delta_{p}$, where $\Delta_{12}=2 \pi \times 3.04 \mathrm{GHz}$ is the hyperfine splitting between $|1\rangle$ and $|2\rangle$. By tuning the pump laser frequency, we are able to generate either degenerate $(\Delta \omega=0)$ or nondegenerate $(\Delta \omega \neq 0)$ photon pairs. We work with a spherical ${ }^{85} \mathrm{Rb}$ cloud with a diameter of $1.4 \mathrm{~mm}$ and temperature of about $100 \mu \mathrm{K}$, trapped by a three-dimensional magneto-optical trap (MOT). The pump and coupling beams, with the same beam diameter of $2 \mathrm{~mm}$, are both linearly polarized along the Stokes-anti-Stokes direction. The phase-matching condition allows generation of backward propagating paired photons in a right-angle geometry [20,21]. As shown in Fig. 1(a), there are two ways to generate the paired photons: (1) The Stokes photon goes to the left and the anti-Stokes to the right, $\left|\omega_{s}\right\rangle_{L}\left|\omega_{\text {as }}\right\rangle_{R}$; and (2) the Stokes photon goes to the right and the anti-Stokes to the left, $\left|\omega_{a s}\right\rangle_{L}\left|\omega_{s}\right\rangle_{R}$. After passing through two polarizers on both sides, the photons are coupled into two opposing polarization-maintaining (PM) single-mode fibers (SMFs). To measure their two-photon interference, the photons are fed into a fiber-coupled BS (OZ Optics, FOBS-22P-PPPP-50/50) whose outputs are directed to two single-photon detectors $D_{1}$ and $D_{2}$ (Perkin Elmer, SPCM-AQ4C). The relative (optical) time delay $\Delta t$ between the two input paths is obtained by using PM SMFs with different lengths. A time-to-digital converter (Fast Comtec TDC 7888) records the coincidence counts with 1-ns time bin. The experiment runs periodically. In each cycle, the MOT trapping time is $4.5 \mathrm{~ms}$, followed by a $0.5-\mathrm{ms}$ biphoton generation window [20]. The trapping magnetic field 

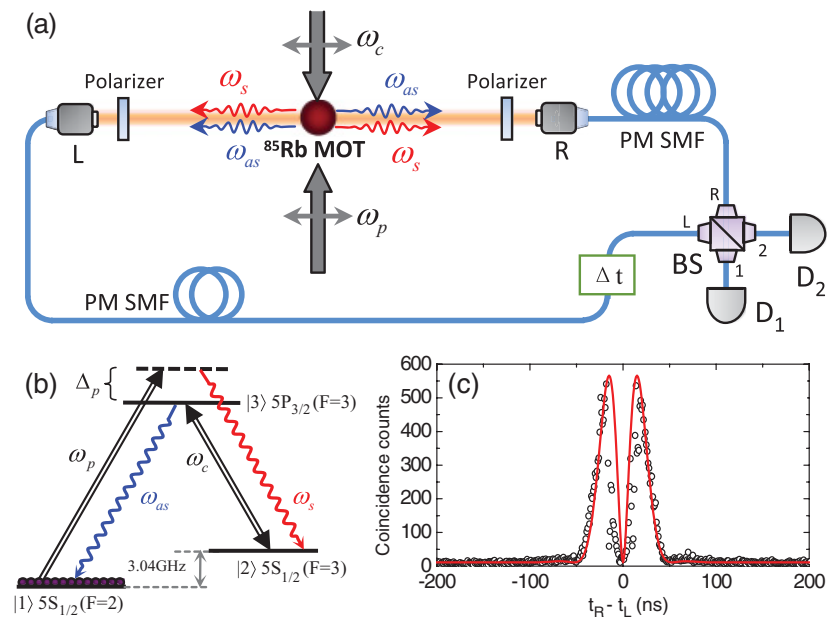

FIG. 1. (Color online) (a) Schematic for paired-photon generation in a right-angle geometry and HOM interference configuration. (b) ${ }^{85} \mathrm{Rb}$ energy-level diagram. (c) Two-photon coincidence counts per 1 -ns bin for $600 \mathrm{~s}$ as a function of the delay time, measured before the beam splitter (BS).

(with a gradient of $5 \mathrm{G} / \mathrm{cm}$ along the Stokes-anti-Stokes direction) remains on always.

In the Schrödinger picture, we have the Stokes and antiStokes biphoton wave function [22]

$$
\begin{aligned}
\Psi_{s, a s}\left(t_{s}, t_{a s}\right) & =\left\langle 0\left|\hat{a}_{a s}\left(t_{a s}\right) \hat{a}_{s}\left(t_{s}\right)\right| \Psi\right\rangle \\
& =e^{-i \omega_{s 0} t_{s}} e^{-i \omega_{a s 0} t_{a s}} \psi_{0}\left(t_{a s}-t_{s}\right),
\end{aligned}
$$

where $\omega_{s 0}$ and $\omega_{a s 0}$ are the central frequencies of Stokes and anti-Stokes photons. With a strong coupling laser and an optical depth of about 5, we work in the damped Rabi oscillation regime [22], where the relative part of the wave function $\psi_{0}(\tau)$ can be approximated as

$$
\psi_{0}(\tau)=B a \sin \left(\frac{\Omega_{c} \tau}{2}\right) e^{-\gamma \tau} \Theta(\tau) .
$$

Here $B$ is a grouped nonlinear coupling coefficient, $a$ is the medium length, $\gamma$ is the damping rate, and $\Theta(\tau)$ is the Heaviside function. Following the right-angle geometry, the biphoton wave packet from the atoms can be described as a superposition

$$
\Psi_{L, R}\left(t_{L}, t_{R}\right)=\Psi_{s, a s}\left(t_{L}, t_{R}\right)+\Psi_{s, a s}\left(t_{R}, t_{L}\right),
$$

which shows the exchange symmetry $\Psi_{L, R}\left(t_{L}, t_{R}\right)=$ $\Psi_{L, R}\left(t_{R}, t_{L}\right)$. Here we denote $L$ and $R$ as the left and right propagation modes as displayed in Fig. 1(a). The Glauber correlation function is $G_{L, R}\left(t_{R}-t_{L}\right)=\left|\Psi_{L, R}\left(t_{L}, t_{R}\right)\right|^{2}=\mid \psi_{0}\left(t_{R}-\right.$ $\left.t_{L}\right)\left.\right|^{2}+\left|\psi_{0}\left(t_{L}-t_{R}\right)\right|^{2}$, which is independent of the central frequencies of the Stokes and anti-Stokes photons. Figure 1(c) shows the two-photon coincidence counts without the BS, which is the same for both degenerate and nondegenerate photons. The theoretical curve is the best fit with $\Omega_{c}=2 \pi \times$ $19 \mathrm{MHz}$ and $\gamma=2 \pi \times 7.64 \mathrm{MHz}$. The right-hand-side wave form is the Stokes-anti-Stokes correlation and the left-hand side is the anti-Stokes-Stokes correlation. The correlation time of about $50 \mathrm{~ns}$ corresponds to a spectral width of $20 \mathrm{MHz}$. Obviously, there is no overlap in time domain between the two terms in Eq. (3) and they do not interfere with each other. It will be shown later that these two terms can interfere after the BS. The nonclassical property of paired photons can be quantified by the violation of the Cauchy-Schwartz inequality [23]. We have the normalized cross-correlation function $g_{s, a s}^{(2)}$ that has a maximum value of 44 . With the autocorrelations $g_{s, s}^{(2)}=g_{\text {as, as }}^{(2)}=2.0$, we violate the inequality $\left[g_{s, a s}^{(2)}\right]^{2} /\left[g_{s, s}^{(2)} g_{a s, a s}^{(2)}\right] \leqslant 1$ by a factor of 484 .

Now we send the photons into the BS, where the field operators take the transformation $\hat{a}_{1}=\left(\hat{a}_{L}-\hat{a}_{R}\right) / \sqrt{2}$ and $\hat{a}_{2}=\left(\hat{a}_{L}+\hat{a}_{R}\right) / \sqrt{2}$. For the relative (optical) time delay $\Delta t=0$, the two-photon wave packet at the two detectors $\left(D_{1}\right.$ and $\left.D_{2}\right)$ can be obtained as $\Psi_{1,2}\left(t_{1}, t_{2}\right)=\left\langle 0\left|\hat{a}_{2}\left(t_{2}\right) \hat{a}_{1}\left(t_{1}\right)\right| \Psi\right\rangle=$ $\left[\Psi_{L, R}\left(t_{1}, t_{2}\right)-\Psi_{L, R}\left(t_{2}, t_{1}\right)\right] / 2=0$. This perfect destructive interference results from the exchange symmetry of the state in Eq. (3), and has nothing to do with the frequency degeneracy of the photons.

An interesting feature emerges when such a symmetry is broken owing to unbalanced paths $(\Delta t \neq 0)$. In this case, the two-photon wave packet becomes $\Psi_{1,2}\left(t_{1}, t_{2}\right)=\left[\Psi_{L, R}\left(t_{1}-\right.\right.$ $\left.\left.\Delta t, t_{2}\right)-\Psi_{L, R}\left(t_{2}-\Delta t, t_{1}\right)\right] / 2$. The relative part of the wave function can be derived as

$$
\begin{aligned}
\psi_{1,2}(\tau, \Delta t)= & \frac{1}{2}\left[\psi_{0}(\tau+\Delta t) e^{-i \Delta \omega(\tau+\Delta t) / 2}\right. \\
& +\psi_{0}(-\tau-\Delta t) e^{i \Delta \omega(\tau+\Delta t) / 2} \\
& -\psi_{0}(\tau-\Delta t) e^{-i \Delta \omega(\tau-\Delta t) / 2} \\
& \left.-\psi_{0}(-\tau+\Delta t) e^{i \Delta \omega(\tau-\Delta t) / 2}\right],
\end{aligned}
$$

where $\tau=t_{2}-t_{1}$ is the relative time delay between the two detectors. With the time bin width of $\delta t=1 \mathrm{~ns}$ that is much shorter than the two-photon correlation time, the two-photon coincidence counts can be expressed as

$$
C_{12}(\tau, \Delta t)=\eta T \delta t\left|\psi_{1,2}(\tau, \Delta t)\right|^{2} .
$$

Here $\eta$ is the two-photon detection efficiency and $T$ is the total time. For the HOM effect, the responses of the slow detectors are modeled as

$$
C_{\mathrm{HOM}}(\Delta t)=\eta T \int_{-W / 2}^{W / 2}\left|\psi_{1,2}(\tau, \Delta t)\right|^{2} d \tau,
$$

where $W$ is the detector time window. For this work, we take $W=100 \mathrm{~ns}$, which is much longer than the two-photon correlation time. Equation (4) suggests that the quantum beat between nondegenerate photons not only can be directly measured by two fast detectors (as a function of $\tau$ ) but also exists in the HOM effect observed with slow detectors (as a function of $\Delta t$ ).

We first confirm the two-photon interference with degenerate photons, i.e., $\omega_{s 0}=\omega_{a s 0}$, by setting the pump detuning as $\Delta_{p}=\Delta_{12}$. The experimental data are displayed in Fig. 2 . When the left and right arms are balanced $(\Delta t=0)$, as expected, we observe a perfect destructive two-photon interference, as shown in Fig. 2(a), where the nonzero background is the accidental coincidence from uncorrelated photons. After passing through the BS, the two indistinguishable photons coalesce and both go to the same output port. When the two arms are unbalanced but the path-length delay difference $(\Delta t=20 \mathrm{~ns})$ is within the coherence time, we observe a partial interference [see Fig. 2(b)]. As $\Delta t$ exceeds the two-photon coherence time, the photons pass through the 

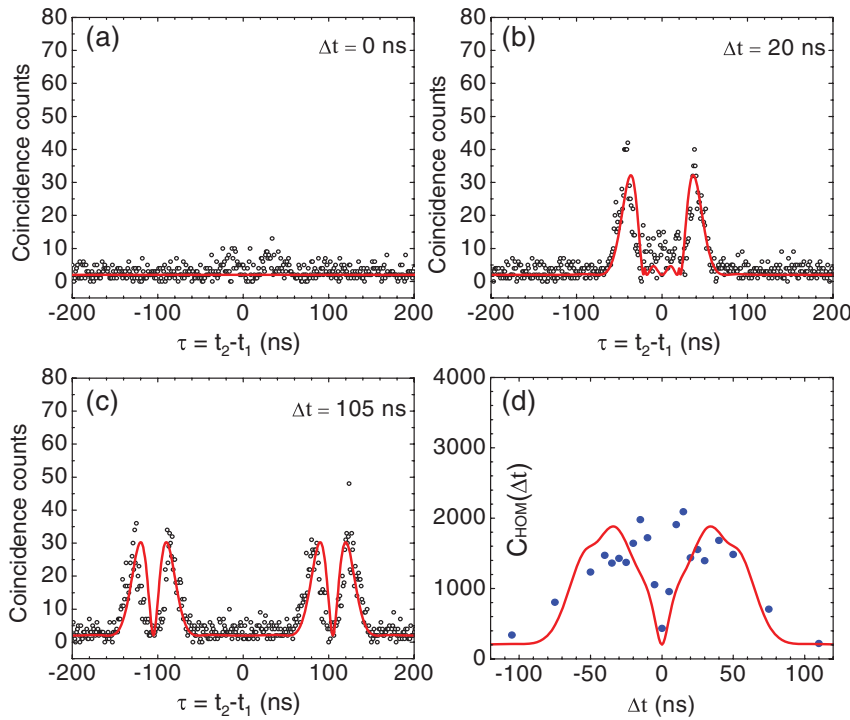

FIG. 2. (Color online) Two-photon interference with degenerate paired photons $(\Delta \omega=0)$. (a)-(c) are the time-resolved two-photon correlations as a function of relative delay between the two detectors $D_{1}$ and $D_{2}$ under different path lengths: (a) $\Delta t=0$, (b) $\Delta t=20 \mathrm{ns,}$ and (c) $\Delta t=105 \mathrm{~ns}$. The coincidence counts are taken in a 1-ns bin for $600 \mathrm{~s}$. (d) HOM coincidence counts as a function of $\Delta t$. The solid curves are plotted from theory.

BS independently and there is no interference, as shown in Fig. 2(c). The HOM coincidence counts as a function of $\Delta t$ are shown in Fig. 2(d) where we integrate the coincidence over $W=100 \mathrm{~ns}$ window. We obtain the theoretical curves (solid lines) from Eqs. (1)-(6), and they agree well with the experimental data. Here we have taken into account the 3-dB fiber-coupling loss at each input port of the BS. The HOM dip has a width of about $50 \mathrm{~ns}$, which is consistent with the direct two-photon correlation measurement as shown in Fig. 1(c). As $\Delta t>\mathrm{W}$, the HOM counts fall back to the background level because the paired photons do not enter the two detectors within the detection time window. The HOM dip has a visibility $V=$ $66 \%$ that exceeds the classical limit of $50 \%$, demonstrating the quantum mechanical origin of the effect [24].

By adjusting the pump laser detuning we produce nondegenerate Stokes and anti-Stokes photon pairs. In the balancedpath configuration $(\Delta t=0)$, as predicted from Eq. (4), the perfect destructive interference always occurs: $\psi_{1,2}(\tau, 0)=0$, because of the symmetry of the input state. This surprising result is confirmed by varying the central-frequency difference between the Stokes and anti-Stokes photons from 0 up to $100 \mathrm{MHz}$ that far exceeds the photon bandwidth of $20 \mathrm{MHz}$. The HOM counts at the dip as a function of frequency difference are shown in Fig. 3. The solid line represents the background accidental coincidence counts from uncorrelated photons. This result suggest that, in our configuration with the symmetry of the input biphoton state, the nondegenerate Stokes and anti-Stokes with different colors interfere and coalesce after the BS. The single photons need not be completely identical (i.e., having different frequencies as in this example) for the HOM effect to occur.

Figure 4 shows the results of the quantum beat experiment when the frequency difference between the Stokes and the

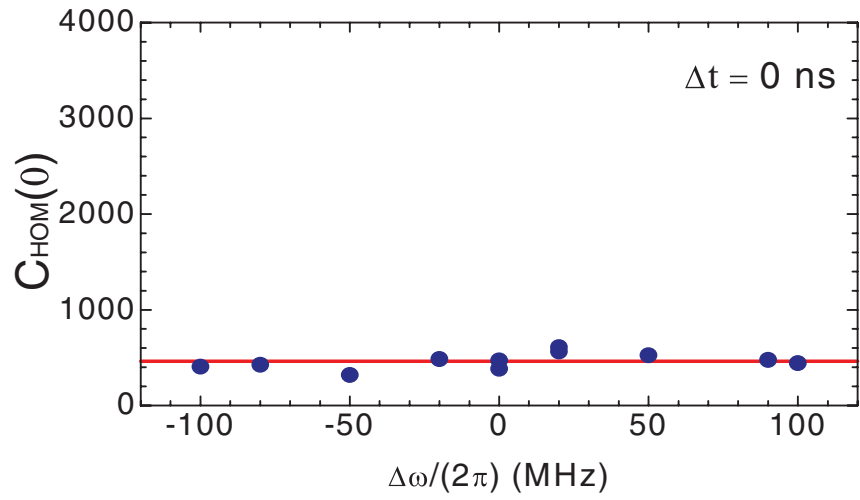

FIG. 3. (Color online) HOM coincidence counts in the balancedpath configuration $(\Delta t=0)$ as a function of frequency difference between the paired photons.

anti-Stokes photons is $\Delta \omega=2 \pi \times 100 \mathrm{MHz}$. At $\Delta t=20 \mathrm{~ns}$, we observe a distinct quantum beat at the coincidence counts by two detectors with a 1-ns bin, as shown in Fig. 4(a). The oscillation period of $10 \mathrm{~ns}$ agrees with the inverse of the photon frequency difference of $100 \mathrm{MHz}$. Figure 4(b) is the HOM coincidence counts $(V=76 \%)$ as a function of $\Delta t$. The data in Fig. 4(b) show that, as predicted from Eqs. (4) and (6), the quantum beat between nondegenerate Stokes and anti-Stokes photons can be resolved by slow detectors. Different from the degenerate case, the dip width here is determined by $2 \pi / \Delta \omega$.
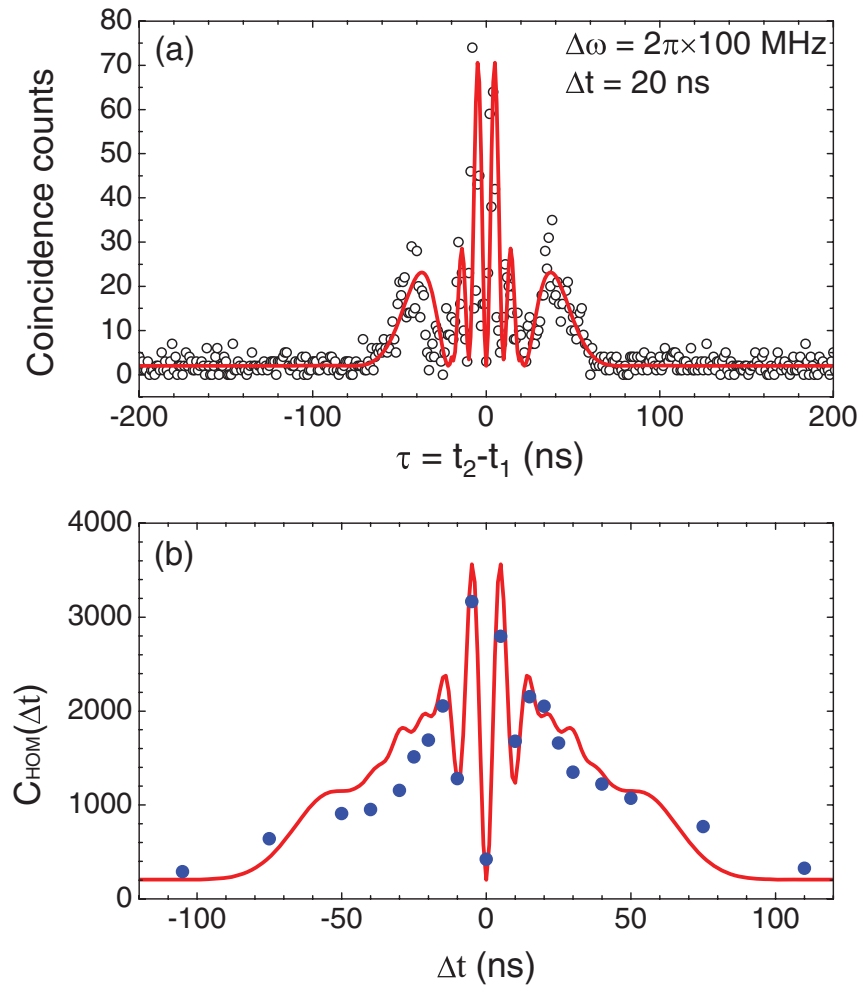

FIG. 4. (Color online) Two-photon interference for the nondegenerate paired photons $(\Delta \omega=2 \pi \times 100 \mathrm{MHz})$. (a) The time-resolved quantum beat: Two-photon coincidence counts as a function of relative delay between the two detectors $D_{1}$ and $D_{2}$ for $\Delta t=20 \mathrm{~ns}$. (b) HOM coincidence counts as a function of $\Delta t$. The solid lines are due to Eqs. (4) and (6). 
The time resolution of our measurement is limited by the discrete fiber length, which cannot be varied continuously. This measurement can be improved if one uses a controllable slow-light system to adjust $\Delta t$.

In summary, we have generated narrow-band paired photons using SFWM with cold atoms in the right-angle geometry and studied their two-photon interference in both degenerate and nondegenerate cases in the HOM setup. When the two paths are balanced, we observe a perfect destructive interference for both degenerate and nondegenerate paired photons. In the degenerate case, the HOM dip resolves the two-photon coherence time of $50 \mathrm{~ns}$, which is consistent with the direct correlation measurement. In the nondegenerate case with a frequency difference up to $100 \mathrm{MHz}$ between the Stokes and anti-Stokes photons, the HOM dip is maintained, but its width is determined by the inverse of the frequency difference. We have also shown that the two slow detectors are able to resolve the quantum beat between the nondegenerate photons using the HOM setup by varying the relative path-length difference, in addition to the direct observation of the quantum beat with fast detectors. Also, when a perfect coalescence interference occurs, the (degenerate or nodegenerate) Stokes and anti-Stokes photons never physically overlap at the beam splitter because the Stokes photon is always produced before the paired anti-Stokes photons-see Fig. 1(c). This shows that the simultaneous arrival of the photons at the beam splitter is not a necessary condition for two-photon interference [10,25].

Our results suggest that exchange symmetry of the photons between the two paths ( $L$ and $R$ spatial modes in our rightangle geometry) is a more general criteria to observe HOM interference, rather than the indistinguishability in photon spectrum. The indistinguishable Feynman pathways in the two-photon probability amplitude caused by this symmetry lead to the HOM effect for both degenerate and nondegenerate paired photons. In our configuration, this symmetry does not depend on the photon frequency degeneracy. When the twophoton amplitude is antisymmetric under the path-exchange operation, the HOM dip becomes a peak $[10,11,13,15]$. Our results may lead to potential applications in linear optical quantum information processing involving photons at different wavelengths.

The work was supported by the Hong Kong Research Grants Council (Project No. 600809).
[1] E. Knill, R. Laflamme, and G. J. Milburn, Nature (London) 409, 46 (2001).

[2] T. B. Pittman, B. C. Jacobs, and J. D. Franson, Johns Hopkins APL Tech. Dig. 25, 84 (2004).

[3] C. K. Hong, Z. Y. Ou, and L. Mandel, Phys. Rev. Lett. 59, 2044 (1987).

[4] L. Mandel, Rev. Mod. Phys. 71, S274 (1999).

[5] K.-I. Harada, H. Takesue, H. Fukuda, T. Tsuchizawa, T. Watanabe, K. Yamada, Y. Tokura, and S.-I. Itabashi, New J. Phys. 13, 065005 (2011).

[6] J. Beugnon, M. P. A. Jones, J. Dingjan, B. Darquié, G. Messin, A. Browaeys, and P. Grangier, Nature (London) 440, 779 (2006).

[7] T. Chaneliere, D. N. Matsukevich, S. D. Jenkins, S.-Y. Lan, R. Zhao, T. A. B. Kennedy, and A. Kuzmich, Phys. Rev. Lett. 98, 113602 (2007).

[8] T. Legero, T. Wilk, A. Kuhn, and G. Rempe, Appl. Phys. B 77, 797 (2003).

[9] T. Legero, T. Wilk, M. Hennrich, G. Rempe, and A. Kuhn, Phys. Rev. Lett. 93, 070503 (2004).

[10] T. B. Pittman, D. V. Strekalov, A. Migdall, M. H. Rubin, A. V. Sergienko, and Y. H. Shih, Phys. Rev. Lett. 77, 1917 (1996).
[11] D. V. Strekalov, T. B. Pittman, and Y. H. Shih, Phys. Rev. A 57, 567 (1998).

[12] A. M. Steinberg, P. G. Kwiat, and R. Y. Chiao, Phys. Rev. A 45, 6659 (1992).

[13] Y.-H. Kim and W. P. Grice, J. Opt. Soc. Am. B 22, 493 (2005).

[14] Z. Y. Ou, Phys. Rev. A 74, 063808 (2006).

[15] K. Wang, J. Phys. B 39, R293 (2006).

[16] Z. Y. Ou and L. Mandel, Phys. Rev. Lett. 61, 54 (1988).

[17] J. G. Rarity and P. R. Tapster, Phys. Rev. A 41, 5139 (1990).

[18] V. Balić, D. A. Braje, P. Kolchin, G. Y. Yin, and S. E. Harris, Phys. Rev. Lett. 94, 183601 (2005).

[19] S. Du, P. Kolchin, C. Belthangady, G. Y. Yin, and S. E. Harris, Phys. Rev. Lett. 100, 183603 (2008).

[20] H. Yan, S. Zhang, J. F. Chen, M. M. T. Loy, G. K. L. Wong, and S. Du, Phys. Rev. Lett. 106, 033601 (2011).

[21] S. Du, E. Oh, J. Wen, and M. H. Rubin, Phys. Rev. A 76, 013803 (2007).

[22] S. Du, J. Wen, and M. H. Rubin, J. Opt. Soc. Am. B 25, C98 (2008).

[23] J. F. Clauser, Phys. Rev. D 9, 853 (1974).

[24] Z. Y. Ou, Phys. Rev. A 37, 1607 (1988).

[25] Y.-H. Kim, Phys. Lett. A 315, 352 (2003). 